liver disease patients (NAFLD) and other 45 cases of newly diagnosed T2DM with NAFLD patients. The physical examination was performed for each patient. Serum levels of alanine aminotransferase (ALT), $\gamma$-glutamyl transpeptidase (GGT), fasting plasma glucose (FPG), lipid profiles, glycation haemoglobin A1c (GHbA1c), creatinine ( $\mathrm{Cr}$ ), uric acid (UA), 2 h postprandic plasma glucose (2hPG), fasting insulin (FINS) were measured. Insulin resistance index (HOMA-IR) was calculated. Tumour necrosis factor- $\alpha$ (TNF $\alpha)$, high sensitive C-reactive protein (hs-CRP) and adiponectin were also detected.

Results The serum levels of ALT and GGT, body mass index and waist hip ratio were higher in the NAFLD, T2DM with NAFLD group patients than that of T2DM and NC group $(p<0.05)$. The serum levels of TG, LDL-C were significantly higher in T2DM, NAFLD and T2DM with NAFLD group patients than that of NC group. And serum TG levels of T2DM with NAFLD were higher than that of T2DM group $(p<0.05)$. FPG and GHbA1c were higher in T2DM and T2DM with NAFLD group patients than that of NAFLD and NC group. The serum levels of TNF $\alpha$ and hs-CRP and HOMA-IR were higher in T2DM, NAFLD and T2DM with NAFLD group patients than that of NC group. And T2DM with NAFLD group patients had higher level of hs-CRP and HOMA-IR compared with T2DM patients. However, serum adiponectin level of T2DM, NAFLD and T2DM with NAFLD group patients were lower than NC group. And it was lower in T2DM with NAFLD group when compared with NC group $(p<0.05)$. There were negative correlations between adiponectin with TNF $\alpha$, hs-CRP and HOMA-IR. But the correlation between HOMA-IR with TNF $\alpha$, hs-CRP was positive.

Conclusions These results suggest that inflammatory factors and adiponectin may play important roles for the pathophysiology and progression of T2DM and NAFLD. The protective effects of adiponectin may lie in anti-inflammation to improve insulin resistant for NAFLD.

\title{
Metabolic syndrome and diabetes
}

\section{[gw22-e0501] CLINICAL RESEARCH FOR THE SIGNIFICANCE OF INFLAMMATORY FACTORS AND ADIPONECTIN IN TYPE 2 DIABETES COMPLICATED WITH NON- ALCOHOLIC FATTY LIVER DISEASE}

Chen Yanming, Wang Manman, He Shengqing, Ren Zhuozhuo, Tang Xixiang, Zeng Longyi Department of Endocrinology, The Third Affiliated Hospital of Sun Yat-Sen University, Guangdong, China

10.1136/heartjnl-2011-300867.305

Objective To investigate the clinical significance of inflammatory factors and adiponectin in type 2 diabetes milletus complicated with non-alcoholic fatty liver disease.

Methods Two hundred and ten subjects aged 25 to 65-yearsold including 106 men and 104 women were recruited into this study. They were divided into four groups: 40 cases of healthy normal control (NC), 60 cases of newly diagnosed type 2 diabetic patients (T2DM), 65 cases of simple non-alcoholic fatty 